\title{
RV Tauri and the RVB phenomenon. I. Photometry of RV Tauri
}

\author{
E. Zsoldos \\ Konkoly Observatory, Budapest XII, P.O.Box 67, 1525 Hungary \\ e-mail: zsoldos@ogyalla.konkoly.hu
}

Received October 4, 1995; accepted February 18, 1996

\begin{abstract}
I present new photoelectric observations of RV Tauri. Period analysis revealed that the amplitude of the long period variation has decreased drastically since the start of the century while the amplitude of the RV Taurilike variation remained practically the same. The change in the short period shows the same kind of cyclic $\mathrm{O}-\mathrm{C}$ diagram as the other variables of this class. While a pulsational origin seems unlikely in the case of the long term variation, its decreasing amplitude indicates that simple binarity could not be used in modelling the RVB phenomena.
\end{abstract}

Key words: stars: individual: RV Tauri — stars: variables — stars: oscillations of — binaries: close

\section{Introduction}

RV Tauri stars form an enigmatic subgroup of pulsating yellow supergiants. Their most characteristic photometric feature is the alternation of shallow and deep minima. Their periods are usually between 50 and 150 days, the visual amplitudes can be sometimes higher than $1^{\mathrm{m}}$. RV Tauri stars have $\mathrm{F}-\mathrm{K}$ spectral types though occasionally one can find examples of earlier types, too (e.g. EP Lyrae).

Though these variables are considered as pulsating stars, the exact mechanism causing the characteristic light variation is still unknown. Basically, there are two competing theories: (1) 2:1 resonance between the fundamental mode and the first overtone (Takeuti \& Petersen 1983, more recently Tuchmann et al. 1993; Fokin 1994); (2) low dimensional chaos (Kovács \& Buchler 1988; Saitou et al. 1989). Since proper RV Tauri models are very scarce, the majority of the theoretical arguments favouring either of the possibilities were based on extrapolation of W Virginis models. The relation of RV Tauri stars and population II Cepheids is, however, not unambiguous so any kind of extrapolation must be viewed with caution. Another obstacle in making reliable RV Tauri models is our lack of knowledge of these stars' basic parameters, e.g. mass, luminosity (Zsoldos 1995).

RV Tauri variables could be divided into two subgroups based on their light curves. The RVA stars have constant mean brightness, typical examples are AC Herculis and EP Lyrae. The RVB stars show a secondary light variation with large amplitude and long period, typical example is RV Tauri (it must be noted here that PayneGaposchkin \& Gaposchkin (1938) denied the RV Tauri status from RV Tauri because of this long-term variation). The distinction between the two subgroups is not clear. Though both RV Tauri and U Monocerotis are classified as RVB their light curves differ significantly. Moreover, the General Catalogue of Variable Stars (GCVS, Kholopov 1985) lists several stars erroneously among the RVBs (e.g. R Sagittae, EP Lyrae).

If the cause of the alternating minima is problematic then the cause of the RVB-like variation is outright mysterious. The only effort to explain these huge variations is that of Fokin $(1988,1994)$, who tried to interpret them as a result of binarity. He, however, used a lot of assumptions not all of which seems necessary.

The light variation of RV Tauri was discovered by L. Ceraski (Ceraski 1905). The star became immediately popular and was instrumental in recognizing that stars like $\mathrm{R}$ Sagittae and V Vulpeculae were not $\beta$ Lyraetype variables but formed a distinct class of their own (Zsoldos 1993b). After the initial burst of interest observations of RV Tauri became rare though fortunately it remained popular with amateur astronomers.

Since RV Tauri has infrared excess (e.g. Jura 1986) it is usually assumed that the star is a post-Asymptotic Giant Branch (post-AGB) object, evolving to higher temperatures to become possibly a planetary nebula. The interpretation of the star's light variation in the context of post-AGB evolution is, however, not clear. 
In this paper (which is part of a series dealing with yellow semiregular variables) I present new photoelectric observations of RV Tauri.

\section{Photometry of RV Tauri}

I observed RV Tauri between 1990 an 1993 in Budapest with the 60-cm telescope of Konkoly Observatory in $B$ and $V$. The proximity of the city made the $U$ observations unreliable. I used $\mathrm{BD}+25^{\circ} 731(V=7 \cdot 71 ; B-V=0.34)$ as comparison star, $\mathrm{BD}+25^{\circ} 734\left(V=8.97 ; B-V=0{ }^{\mathrm{m}} 53\right)$ was the check star (Dawson \& Patterson 1982). The observations are listed in Table 1 . The average errors in $V$ are about $0{ }^{\mathrm{m}} 01$, in $B-V$ they are about 0.015 .

Table 1. Observations of RV Tauri

\begin{tabular}{crr}
\hline J.D. & $V$ & $B-V$ \\
$2400000.0+$ & & \\
\hline 48163.582 & 9.284 & 1.479 \\
48176.520 & 9.493 & 1.770 \\
48186.602 & 10.145 & 1.740 \\
48187.594 & 10.366 & 1.717 \\
48190.547 & 10.406 & 1.781 \\
48197.527 & 9.633 & 1.400 \\
48202.492 & 9.189 & 1.432 \\
48212.492 & 9.170 & 1.550 \\
48271.332 & 10.048 & 1.602 \\
48288.313 & 9.042 & 1.694 \\
48305.258 & 9.476 & 1.650 \\
48534.547 & 9.537 & 1.981 \\
48561.488 & 8.916 & 1.656 \\
48562.508 & 8.932 & 1.692 \\
48573.500 & 9.490 & 1.876 \\
48592.355 & 9.116 & 1.463 \\
48593.422 & 9.104 & 1.491 \\
48600.391 & 9.113 & 1.662 \\
48603.395 & 9.183 & 1.711 \\
48642.313 & 9.261 & 1.771 \\
48646.324 & 9.385 & 1.863 \\
49005.371 & 9.749 & 1.728 \\
49006.371 & 9.774 & 1.829 \\
\hline & &
\end{tabular}

The light and colour curves of the variable are plotted in Fig. 1. It shows a well developed alternation confirming the "RV Tauri" nature of RV Tauri. The phase lag between the $V$ and $B-V$ maximum is clearly seen, the maximum in $B-V$ occuring earlier than in $V$ similarly to other RV Tauri variables (e.g. EP Lyrae, see Zsoldos 1995).

It is interesting to note that though my observations cover almost 900 days, there is no trace of the long period variation. This is quite surprising considering the RVB nature of the star. It is, however, confirmed by the analysis of the available data (see next section).

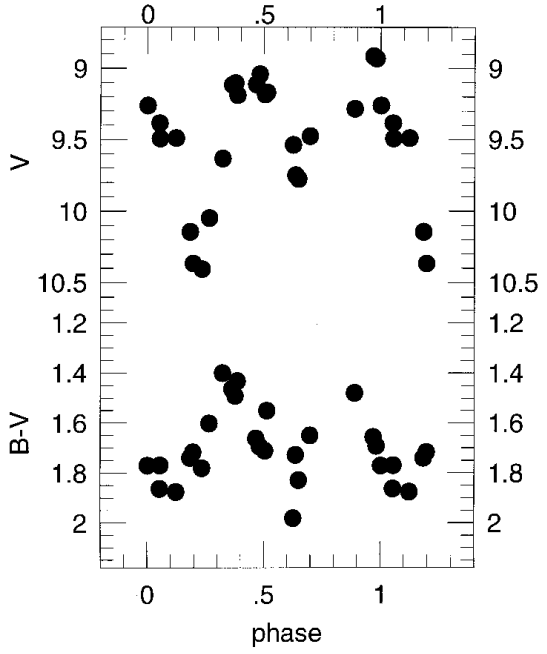

Fig. 1. The light and colour curves of RV Tauri

\section{Period analysis}

Unfortunately, there are very few photoelectric observations of RV Tauri. $U B V$ photometry has been published by DuPuy (1973), Dawson (1979), Dawson \& Patterson (1982) and Wahlgren (1992). Photographic observations were given by Payne-Gaposchkin et al. (1943) and Rosino (1951) while visual observations by Beyer (1930), Ceraski (1905), Enebo (1907), Hartwig (1913), McLaughlin (1934), Pračka (1910), Seares \& Haynes (1908) and Stein (1944). Van der Bilt (1916) collected all visual observations of his time including a large number of unpublished ones. I also used the data collected by the AFOEV (Schweitzer \& Vialle 1993). I used the MUFRAN package (Kolláth 1990) for period analysis which is based on the Deeming method.

Since the photoelectric observations are few and far between they are not well suited for period analysis. The only frequency it shows with any certainty (see Fig. 2) is at $f=0.0254486(P=39.29)$ with an amplitude of 0.44 . It should be noted that, in accordance with my observations, there is no clear sign of a long period variation. Moreover, though Fig. 1 clearly shows the presence of the alternating minima, there is no sign of the fundamental period either. Its cause is probably the very poor phase coverage of the individual observing runs.

The photographic and visual observations are fortunately more numerous. The two sets gave very similar results (compare Figs. 3 and 4). Since the visual observations cover a longer period of time I will discuss only their analysis.

There are two groups of frequencies: one corresponding to the long period variations (around the frequency $f_{1}=0.0008311$ ), the other to the RV Tauri-like variations ( $f=0.025421$ and related frequencies). In Table 2 one can compare the frequencies and their amplitudes in two well 

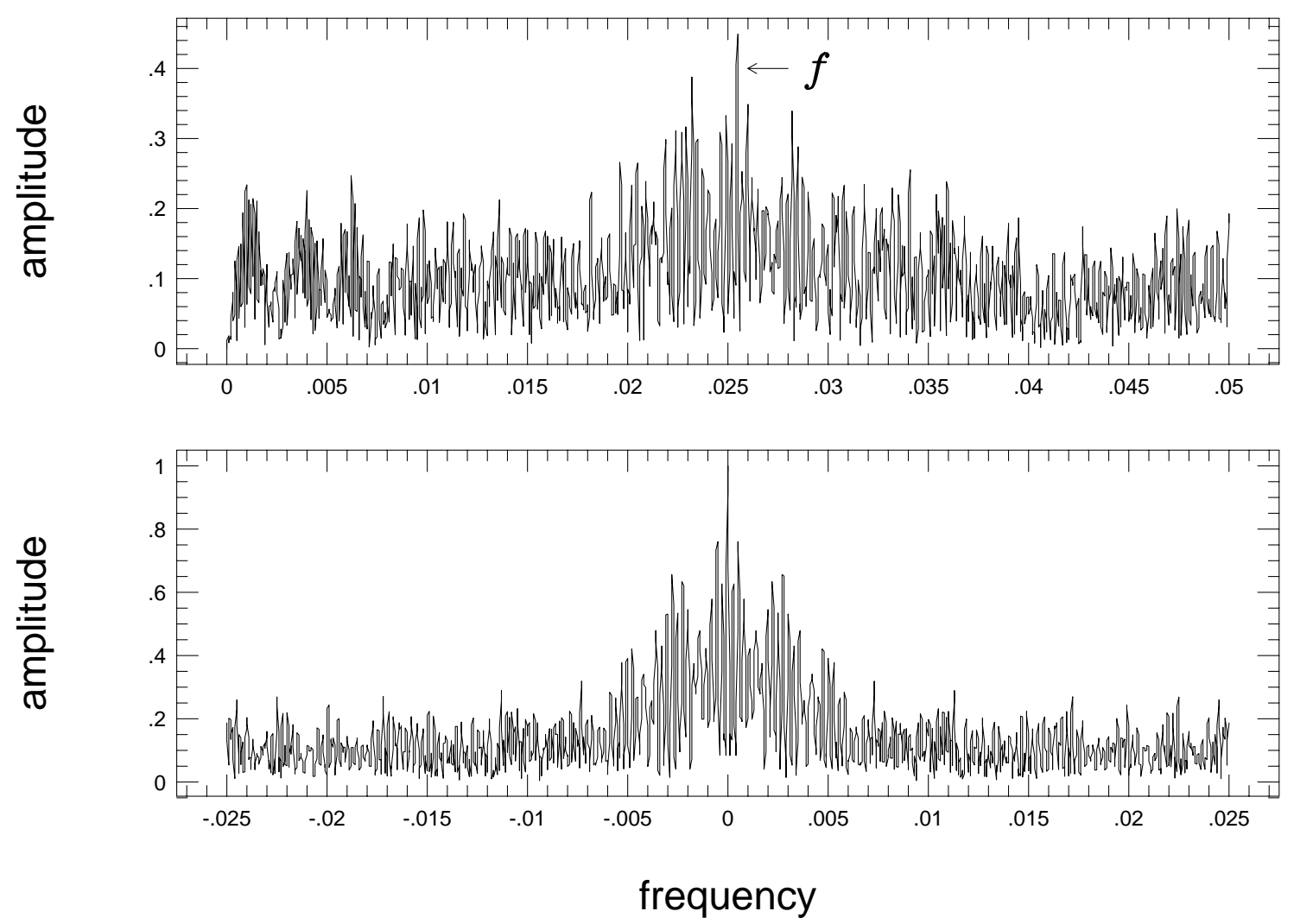

Fig. 2. The power spectrum and spectral window of the photoelectric observations of RV Tauri. The observations were made between 1970 and 1993. The peak, indicated by an arrow, corresponds to the 'half-period' $(f)$

observed periods, $1925-40$ and $1980-95$ (this is by far the best observed part).

Table 2. Comparison of frequencies

\begin{tabular}{llllr}
\hline \multicolumn{2}{c}{$1925-40$} & \multicolumn{2}{c}{$1980-95$} & \\
freq. & ampl. & freq. & ampl. & interpretation \\
\hline 0.025417 & 0.37 & 0.025421 & 0.40 & $2 f_{0}$ \\
0.000840 & 0.61 & 0.000856 & 0.21 & $f_{1}^{\mathrm{m}} 21$ \\
0.012752 & 0.09 & 0.012727 & $0 .{ }^{\mathrm{m}} 12$ & $f_{0}$ \\
& & 0.050842 & $0 .{ }^{\mathrm{m}} 06$ & $4 f_{0}$ \\
0.001667 & 0.21 & & & $2 f_{1}$ \\
0.002509 & 0.12 & & & $3 f_{1}$ \\
0.000184 & 0.36 & & & $?$ \\
\hline
\end{tabular}

There are several interesting characteristics of the variation of RV Tauri which are immediately recognizable from Table 2 and Figs. 2-4:

- While the amplitude of the RV Tauri-like light curve is practically the same, there is a drastic decrease in the amplitude of the long-term variation.

- The short and long periods show only a very slight variation $\left(78^{\mathrm{d}} .42 ; 78^{\mathrm{d}} .57\right.$ and $1190^{\mathrm{d}} ; 1168^{\mathrm{d}}$, respectively).
Section 4 will deal with period variations in more detail.

- All the other periods seem to be harmonics of either $f_{0}$ or $f_{1}$. The exception is $f=0.000184$ which might be the result of some hazards of the visual observations (e.g. different zero points, different magnitude values of comparisons, etc.).

- The ratio of the amplitudes of $f_{0}$ and $2 f_{0}$ seems very low (0.24-0.30) compared to other RV Tauri stars (e.g. EP Lyrae: 1.02 (Zsoldos 1995), AC Herculis: 0.50, R Sagittae: 0.50, V Vulpeculae: 1.05 (Zsoldos 1993a)).

Since the nature of the long term variation is unknown its apparent amplitude variation might be very important. The visual observations were divided into ten years long parts and each was fitted with the frequencies in Col. 3 of Table 2 (since this was the best observed part of the light curve I consider these frequencies to be the most reliable). Table 3 shows the amplitude variations between 1900 and 1995.

It is clear from Table 3 that $3 f_{0}$ and $4 f_{0}$ have only rarely noticeable amplitudes so their presence in the light curve is certainly dubious.

Since the above division of the light curve is rather arbitrary, the fit was performed again by starting at 1923 and shifting the ten-year long interval by one year 

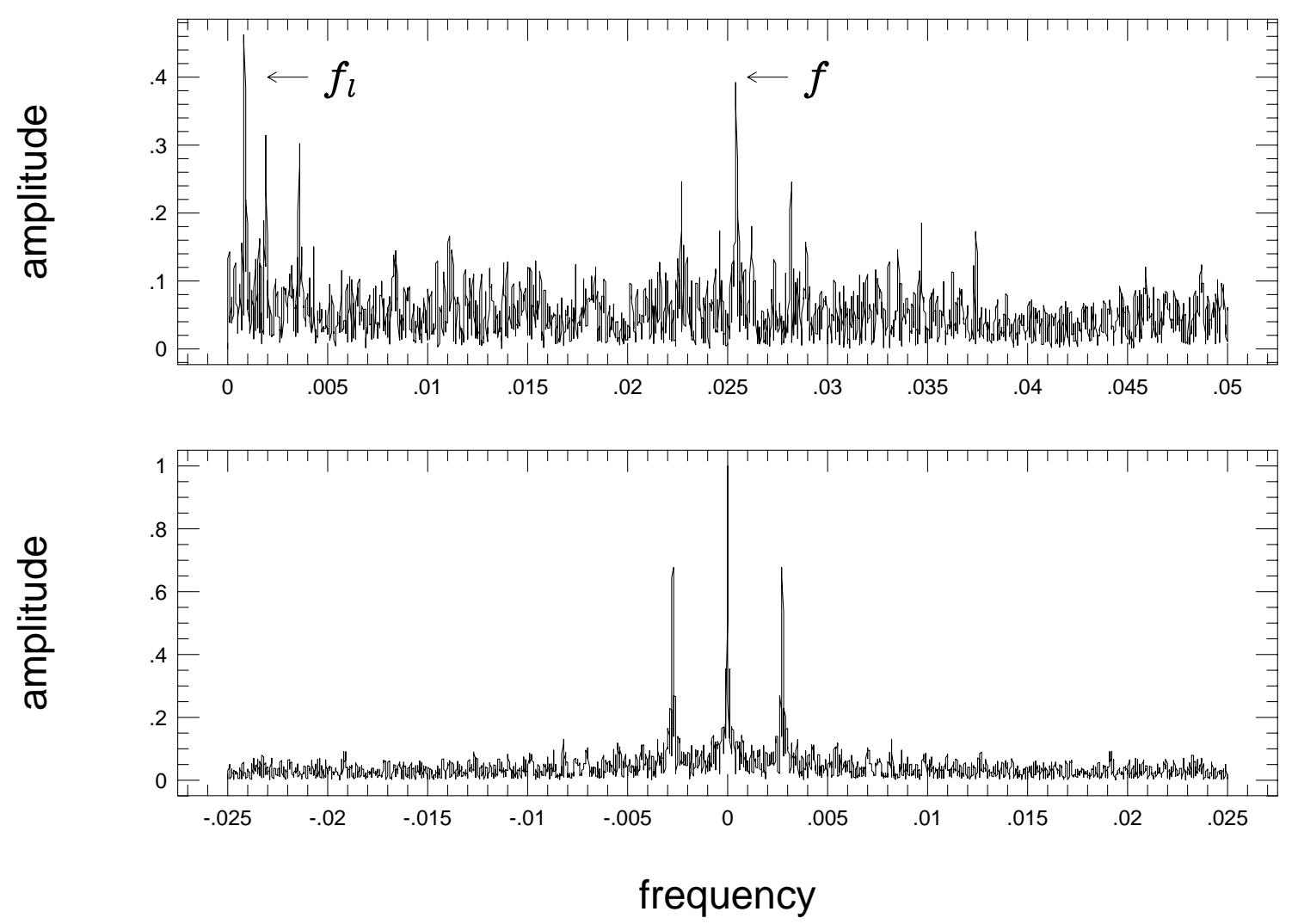

Fig. 3. The power spectrum and spectral window of the photographic observations of RV Tauri. The observations were made between 1898 and 1950. The two peaks correspond to the long period variation $\left(f_{1}\right)$ and the 'half-period' $(f)$, respectively

Table 3. Amplitudes of the various frequencies

\begin{tabular}{|c|c|c|c|c|c|c|}
\hline decade & $f_{0}$ & $2 f_{0}$ & $3 f_{0}$ & $4 f_{0}$ & $f_{1}$ & s.d.fit \\
\hline $1901-10$ & $0 . \mathrm{m} 15$ & m 37 & ${ }^{\mathrm{m}} 04$ & m 08 & & \\
\hline & & & & ${ }^{\mathrm{m}} 05$ & $\mathrm{~m}^{\mathrm{m}} 23$ & $\mathrm{~m}^{\mathrm{m}} 22$ \\
\hline & & & & $\mathrm{m} 06$ & & 0.26 \\
\hline & 0.05 & 0.35 & m 02 & m 04 & 0.58 & 0.32 \\
\hline & & & & & & $0 \cdot 34$ \\
\hline & $0{ }^{\mathrm{m}} 04$ & & $0{ }^{\mathrm{m}} 06$ & 0.07 & 0.13 & 0.30 \\
\hline & & & $0{ }^{\mathrm{m}} 03$ & $0 .{ }^{\mathrm{m}} 06$ & $0 .{ }^{\mathrm{m}} 18$ & 0.27 \\
\hline $1991-95$ & $0{ }^{\mathrm{m}} 11$ & $00^{\mathrm{m}} 41$ & $0{ }^{\mathrm{m}} 03$ & 0.08 & 0.26 & $0 .{ }^{\mathrm{m}} 24$ \\
\hline
\end{tabular}

continuously. Since there are no observations available from 1945-47 the intervals containing these years have lower weight. The error of the amplitudes is less than $0{ }^{\mathrm{m}} 1$ even in the most uncertain cases. Using the same frequencies as in Table 3 , the amplitude variation of $f_{1}$ was fully confirmed (see Fig. 5). It seems that the change occured rather abruptly around the early 1940's and the amplitude remained practically constant after the drop (though one must not forget the gap in Fig. 5 due to the lack of data between 1953 and 1966). Since the difference between the pre-1940 and post-1940 amplitudes is quite large, it is probably safe to assume that it represents a real change.

\section{Period change}

Though the period of RV Tauri seems to be rather stable it is worth constructing its $\mathrm{O}-\mathrm{C}$ diagram. It was previously investigated e.g. by Tessevich (1951) and Isles (1975), the latter found the period of the star practically constant.

The majority of the minima were collected from the literature, three further minima were estimated from the photoelectric light curve. Van der Bilt (1916) gathered all the then available minima similarly to the observations. They were also published by Beyer (1930), Huth (1964), Isles (1975), Jacchia (1929, 1930, 1931), Lause (1930, 1931, 1934), McLaughlin (1934) and Nijland (1910, 1911, 1912, 1913, 1914, 1915a, 1915b, 1918, 1921, 1922a, 1922b, 1923, 1924, 1925, 1926, 1927, 1928, 1929, 1930, 1931, 1932, 1933, 1934, 1936).

Figure 6 shows the $\mathrm{O}-\mathrm{C}$ diagram of RV Tauri using an average period of 78.74 and an arbitrary epoch of 2400060.0. There is a clear though slight period change, displaying the same wavy character that seems to be usual in RV Tauri variables (see e.g. Zsoldos 1991, 1993a, 1995; Percy et al. 1992). The interval between the two 'minima' 

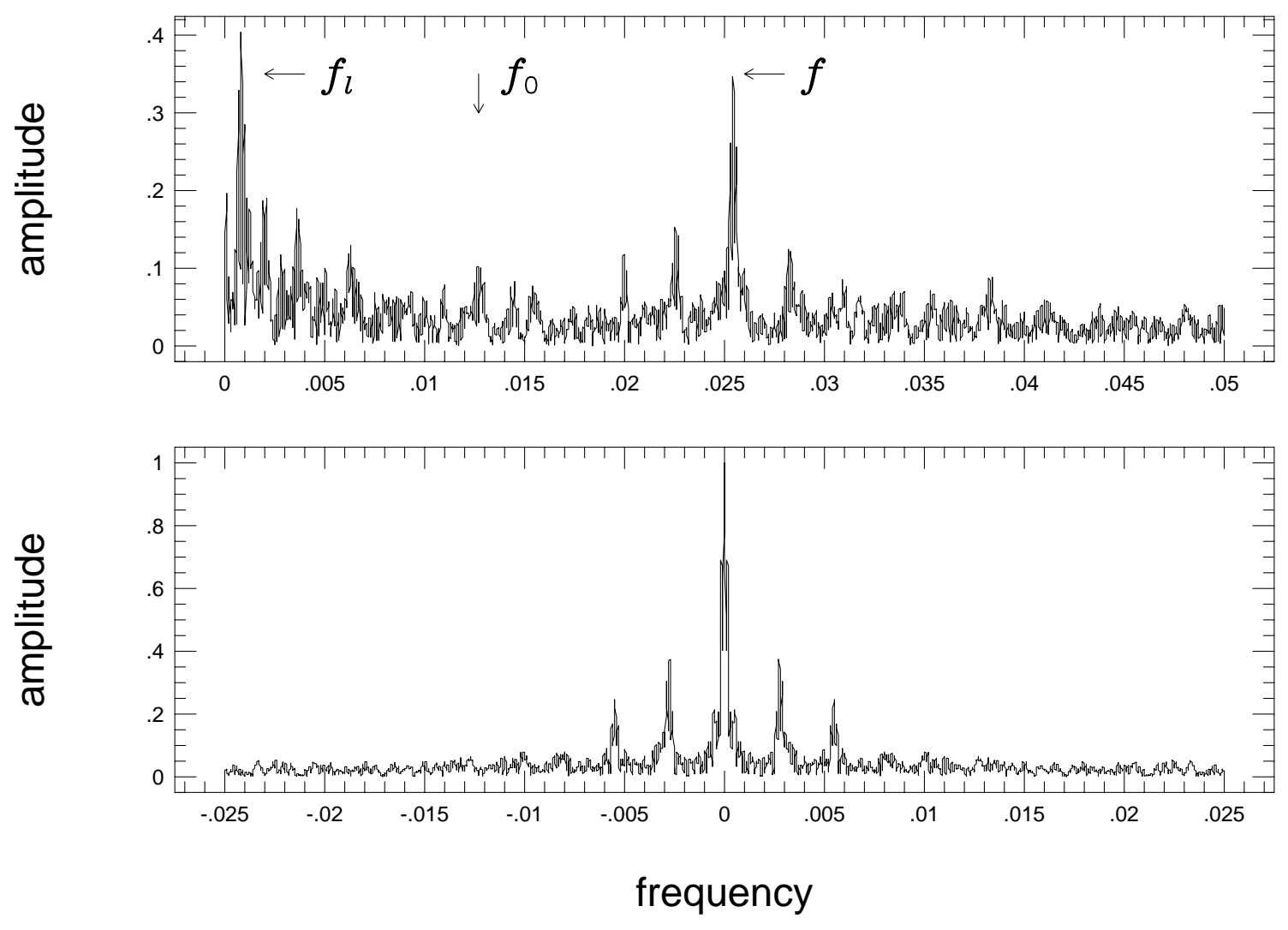

Fig. 4. The power spectrum and spectral window of the visual observations of RV Tauri. The observations were made between 1895 and 1995. The arrows point to $f_{1}$ (the long period), $f_{0}$ (the formal period) and $f$ (the half-period)

in the diagram is about 20000 days and the 'amplitude' of the cycle is about 9 days. With $(B-V)_{0}=1^{\mathrm{m}} \cdot 16$ (see next section) RV Tauri certainly does not fit the suggested relation between colour and amplitude of $\mathrm{O}-\mathrm{C}$ cycle (Zsoldos 1995). One must note, however, that this relation was found for RVA stars, i.e. for variables with constant mean brightness.

The origin of this kind of $\mathrm{O}-\mathrm{C}$ diagram is not clear. RV Tauri itself, however, fits the Eddington-Plakidis (Eddington \& Plakidis 1929) model for random period fluctuations very well (Percy et al. 1996).

\section{Discussion}

\subsection{The properties of $R V$ Tauri}

As with the other RV Tauri variables, the physical parameters of RV Tauri itself are very poorly determined. Its spectral type is usually given as G2-K3 I (Rosino 1951; Joy 1952) the only exception is an M2 Ia type observed at Jan. 24, 1962 by Preston et al. (1963). This date, however, belongs to a minimum.

The colour excess of RV Tauri is given in the literature as 0.41 (DuPuy 1973), 0.54 (Straižys \& Meištas 1981) and 0.07 (Wahlgren 1992). The reddening maps of Burstein \& Heiles (1982) support an $E(B-V) \approx 0.5$ in good agreement with all determinations but Wahlgren's which was made near a minimum. Accepting $0 \stackrel{\mathrm{m}}{50}$ as the colour excess results in $(B-V)_{0}=1 .{ }^{\mathrm{m}} 16$ which corresponds to a spectral type of G8 or K0, depending on the population type of RV Tauri (Schmidt-Kaler 1982; Osborn 1973). This spectral type implies an effective temperature of $4600 \mathrm{~K}$, in fairly good agreement with the value of $4250 \mathrm{~K}$ derived by Wahlgren (1992). Since the [Fe/H] values given in the literature are -0.63 (Dawson 1979) and -1.0 (Wahlgren 1992) the star seems to belong to population II.

The only mass determination is that of Dawson (1979), he gave $6.5 M_{\odot}$, about ten times higher than the usually assumed post-AGB mass.

\subsection{The long period}

The long period variation leads to several interesting problems. It has the following properties (see e.g. Fokin 1994; Pollard et al. 1996):

- There is a relation between the short (formal) and the long period (O'Connell 1933; Tsessevich 1933; Parenago 1937).

- The RV Tauri-like variation has a smaller amplitude when the long period change is in minimum. 
Fokin (1994) tried to explain the phenomenon as a result of binarity though the mechanism he suggested did not require a companion (eclipse by circumstellar material). Eclipse by a companion seems to be ruled out by the drastic amplitude change as it would probably require some very special and quick change in the geometry of the system.

The second property (smaller amplitudes when the long term variation is in minimum) is somewhat uncertain. The visual observations in fact indicate an amplitude change in the RV Tauri-like part of the variation. There is another frequency, $f^{\prime}=0.0247286$ besides $2 f_{0}=0.025432$ but the frequency of the change these close periods imply $\left(\Delta f=2 f_{0}-f^{\prime}=0.000703\right)$ is different from $f_{1}(=0.0008311)$. Moreover, $f^{\prime}$ might not be a real frequency since it is not present either in the $1925-40$ or the 1980-95 sets.

It seems therefore that the connection between $f_{0}$ and $f_{1}$, at least in the case of RV Tauri, is not proved. Though it is possible to suppose that both come from pulsation (e.g. Tsessevich 1957), the period ratio $P_{0} / P_{1}$ would be $\approx 15$ which is far too high (Fox \& Wood 1982 had models with a ratio as high as 7 in some cases, but their models referred to high mass stars) making the assumption untenable. One should note, however, that the GCVS lists several SRA and SRB stars with period ratios larger than ten, e.g. LU Aquilae $\left(P / P_{1}=10.6\right)$ or RV Aurigae $\left(P / P_{1}=45.4\right.$ !).

There are some other possibilities to explain the RVB phenomenon. The first is to suppose that it is really a binary, and the two kinds of variation come from the two stars. The second is a kind of envelope activity (with or without binarity) - somewhat similar to the RCB variables - originally suggested by Percy (binary, 1993) and Shenton et al. (non-binary, 1994) for the case of U Monocerotis. The latter might be helpful explaining the decreasing amplitude of the long period variation. A variant of the binary model was suggested recently by Pollard et al. (1996) in which interaction between the variable and its companion might play a crucial role.

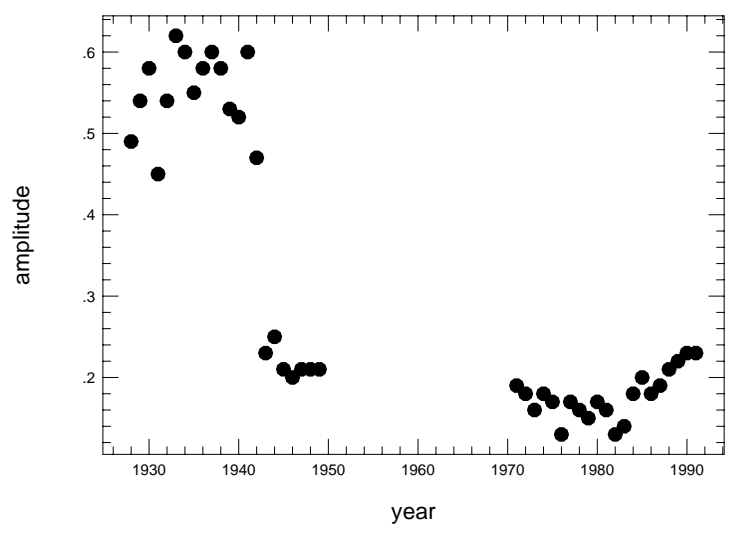

Fig. 5. The amplitude variation of the long period

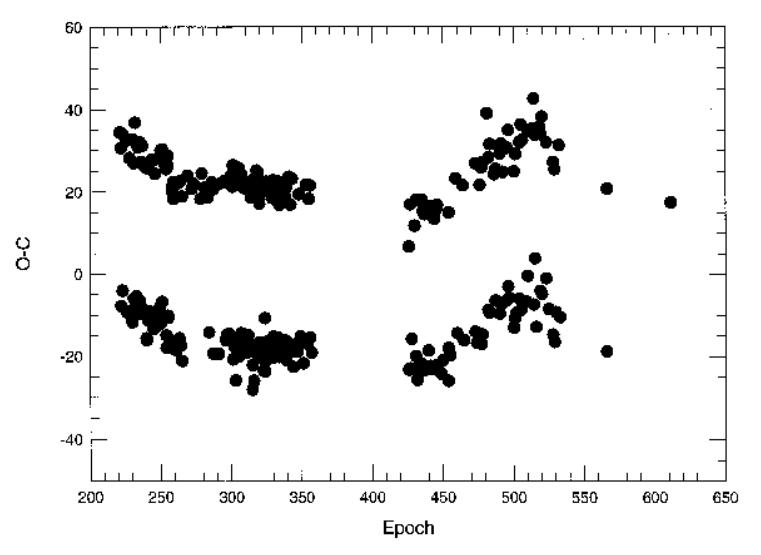

Fig. 6. The $\mathrm{O}-\mathrm{C}$ diagram of RV Tauri. The upper plot is for the primary, the lower is for the secondary minima

\section{Conclusions}

New photometry of RV Tauri clearly shows the presence of alternating deep and shallow minima thus confirming the inclusion of the star among the RV Tauri variables (this classification was questioned by Payne-Gaposchkin \& Gaposchkin 1938). The period of the variable shows only slight changes, its $\mathrm{O}-\mathrm{C}$ diagram displays the characteristic wavy structure.

The long period variation offers several interesting features. Contrary to general belief the amplitude of the short period variation does not necessarily decrease when the long term variation is in minimum. More interesting is the decrease of the amplitude of the secondary variation. This apparently secular change might indicate that the origin of the RVB phenomenon might not be binarity for which there is no evidence - but some kind of envelope activity.

Acknowledgements. I wish to thank Béla Szeidl for the stimulating discussions and John Percy for his valuable comments. The helpful comments of the referee, Dr. M.O. Mennessier, are also acknowledged. This research was partially supported by the OTKA grants T-014183 and T-015759.

\section{References}

Beyer M., 1930, Astr. Abh. 8, No. 3

Burstein D., Heiles C., 1982, AJ 87, 1165

Ceraski W., 1905, Astron. Nachr. 168, 29

Dawson D.W., 1979, ApJS 41, 97

Dawson D.W., Patterson C.R., 1982, PASP 94, 574

DuPuy D.L., 1973, ApJ 185, 597

Eddington A.S., Plakidis S., 1929, MNRAS 90, 65

Enebo S., 1907, Astron. Nachr. 175, 205

Fokin A.B., 1988, Nauchn. inf. No. 65, 64

Fokin A.B., 1994, A\&A 292, 133

Fox M.W., Wood P.R., 1982, ApJ 259, 198

Hartwig E., 1913, Bamberg Veröff. 1, No. 2

Huth H., 1964, Mitt. Veränd. Sterne 2, 112 
Isles J.E., 1975, J. British Astron. Assoc. 85, 156

Jacchia L., 1929, Beob. Zirk. 11, 7, 24, 28, 89

Jacchia L., 1930, Beob. Zirk. 12, 3, 16, 54

Jacchia L., 1931, Beob. Zirk. 13, 16

Joy A.H., 1952, ApJ 115, 25

Jura M., 1986, ApJ 309, 732

Kholopov P.N., 1985, General Catalogue of Variable Stars, 4th ed., Nauka Publ., Moscow

Kolláth Z., 1990, Konkoly Obs. Occasional Techn. Notes, No. 1

Kovács G., Buchler J.R., 1988, ApJ 334, 971

Lause F., 1930, Astron. Nachr. 239, 59

Lause F., 1931, Astron. Nachr. 244, 79

Lause F., 1934, Astron. Nachr. 251, 43

McLaughlin D.B., 1934, AJ 44, 41

Nijland A.A., 1910, Astron. Nachr. 184, 193

Nijland A.A., 1911, Astron. Nachr. 187, 401

Nijland A.A., 1912, Astron. Nachr. 190, 433

Nijland A.A., 1913, Astron. Nachr. 194, 183

Nijland A.A., 1914, Astron. Nachr. 199, 209

Nijland A.A., 1915a, Astron. Nachr. 200, 353

Nijland A.A., 1915b, Astron. Nachr. 203, 133

Nijland A.A., 1918, Astron. Nachr. 206, 173

Nijland A.A., 1921, Astron. Nachr. 212, 493

Nijland A.A., 1922a, Astron. Nachr. 215, 361

Nijland A.A., 1922b, Astron. Nachr. 217, 7

Nijland A.A., 1923, Astron. Nachr. 219, 333

Nijland A.A., 1924, Astron. Nachr. 221, 213

Nijland A.A., 1925, Astron. Nachr. 224, 213

Nijland A.A., 1926, Astron. Nachr. 227, 97

Nijland A.A., 1927, Astron. Nachr. 229, 345

Nijland A.A., 1928, Astron. Nachr. 233, 297

Nijland A.A., 1929, Astron. Nachr. 235, 291

Nijland A.A., 1930, Astron. Nachr. 238, 237

Nijland A.A., 1931, Astron. Nachr. 242, 23

Nijland A.A., 1932, Astron. Nachr. 245, 149

Nijland A.A., 1933, Astron. Nachr. 249, 1

Nijland A.A., 1934, Astron. Nachr. 252, 69

Nijland A.A., 1936, Astron. Nachr. 259, 69

O'Connell D.J.K., 1933, Harvard Bull. 893, 19

Osborn W., 1973, in: Fehrenbach C., Westerlund B.E. (eds.), IAU Symp. No. 50, Spectral Classification and Multicolour
Photometry. Reidel, Dordrecht, p. 176

Parenago P.P., 1937, Perem. Zv. 5, 149

Payne-Gaposchkin C., Gaposchkin S., 1938, Variable Stars, Harvard Observatory Monographs No. 5, Harvard Observatory, Cambridge, Mass

Payne-Gaposchkin C., Brenton V.K., Gaposchkin S., 1943, Harvard Ann. 113, No. 1

Percy J.R., 1993, in: Sasselov D.D. (ed.), Luminous HighLatitude Stars, ASP Conf. No. 45, p. 295

Percy J.R., Csatary A., Zorgdrager I., 1992, J. AAVSO 21, 86

Percy J.R., Zsoldos E., Bezuhly M., Milanowski M., 1996, (in preparation)

Pollard K.R., Cottrell P.L., Kilmartin P.M., Gilmore A.C., 1996, MNRAS (in press)

Pračka L., 1910, Bericht Astrophys. Obs. Nižbor 1, No. 2

Preston G.W., Krzeminski W., Smak J., Williams J.A., 1963, ApJ 137, 401

Rosino L., 1951, ApJ 113, 60

Saitou M., Takeuti M., Tanaka Y., 1989, PASJ 41, 297

Schmidt-Kaler T., 1982, Landolt-Börnstein, New Series, Group VI, Vol. 2b, p. 1

Schweitzer E., Vialle J., 1993, AFOEV Bull. No. 64, p. 13

Seares F.H., Haynes E.S., 1908, Laws Obs. Bull. No. 14

Shenton M., Evans A., Cardelli J.A., Marang F., van Wyk F., Weight A., 1994, A\&A 287, 852

Stein J., 1944, Ric. Astron. Specola Vaticana 1, 301

Straižys V., Meištas E., 1981, AZh 58, 367

Takeuti M., Petersen J.O., 1983, A\&A 117, 352

Tsessevich V., 1933, Bull. Leningrad Univ. Astron. Obs. No. 2 , p. 2

Tsessevich V.P., 1951, Perem. Zv. 8, 121

Tsessevich V.P., 1957, in: Herbig G.H. (ed.), IAU Symp. No. 3, Non-Stable Stars. Cambridge Univ. Press, p. 116

Tuchman Y., Lèbre A., Mennessier M.O., Yarri A., 1993, A\&A 271,501

van der Bilt J., 1916, Utrecht Res. Vol. 6

Wahlgren G.M., 1992, AJ 104, 1174

Zsoldos E., 1991, Ap\&SS 181, 203

Zsoldos E., 1993a, A\&A 268, 149

Zsoldos E., 1993b, The Observatory 113, 305

Zsoldos E., 1995, A\&A 296, 122 